\title{
Qual Guia de Comunicação na Consulta Médica É o Mais Adequado para o Ensino de Habilidades Comunicacionais na Atenção Primária à Saúde Brasileira?
}

\author{
Which Communication Guide for Medical \\ Visits is the Most Appropriate for Teaching \\ Communication Skills in Primary Health Care in \\ Brazil?
}

\section{PALAVRAS-CHAVE}

- Comunicação em Saúde.

- Atenção Primária à Saúde.

- Materiais de Ensino.
Carlos Frederico Confort Campos ${ }^{I}$ Izabel Cristina Rios ${ }^{I I I I I}$

\begin{abstract}
RESUMO
Introdução: $O$ uso de habilidades de comunicação está associado a desfechos positivos na consulta médica e no cuidado à saúde, como melhora da adesão aos tratamentos, da satisfação de médicos e pacientes, e da relação entre eles. Isto é ainda mais significativo na Atenção Primária à Saúde (APS), que é um espaço de cuidado integral de saúde. Uma comunicação efetiva é aquela em que todos os participantes interagem de modo a trocar informações e em que, ao final, todas as partes tenham o mesmo entendimento sobre o problema, acordem sobre as medidas a tomar e se sintam entendidas em suas necessidades. Para que este tipo de comunicação aconteça, o ensino dessas habilidades deve ocorrer de forma adequada e contínua, durante toda a formação médica. Até o momento, não existe um instrumento brasileiro que cumpra esta finalidade. No Brasil, têm-se utilizado guias internacionais para este ensino. No entanto, dos guias mais usados internacionalmente e mais citados na literatura da área, nenhum é completamente adequado à realidade da APS brasileira. Objetivo: Comparar quatro instrumentos de habilidades de comunicação frequentemente utilizados, tendo em vista os aspectos que caracterizam a APS nacional. Metodologia: Os guias foram descritos, analisados qualitativamente e comparados quanto às seguintes categorias: estrutura do guia, criação do guia, etapas importantes da consulta e foco no médico. Análise: Os instrumentos diferem quanto a facilidade de uso, foco no ensino de APS, discussão de etapas importantes da consulta e foco no médico; todos discutem a construção de planos compartilhados e nenhum tem uma tradução validada para português do Brasil. Conclusão: Dos quatro guias estudados, "A Consulta em 7 em Passos" tem o melhor perfil de uso para a APS nacional. No entanto, recomenda-se a criação de um guia brasileiro para estruturar, homogeneizar e facilitar o ensino das habilidades de comunicação nas escolas médicas do País.
\end{abstract}




\section{KEY-WORDS}

- Health Communication.

- Primary Health Care.

- Teaching Materials.

Recebido em 28/11/2017

Aceito em 12/03/2018
ABSTRACT

Introduction: the use of communication skills is associated with positive outcomes in medical visits and health care, such as better adherence to treatments, satisfaction of doctors and patients and the relationship between them. This is even more significant in Primary Health Care, which is a space of comprehensive health care. An effective communication happens whenever all participants interact to exchange information and, in the end, everyone has the same understanding about the problem, everyone agrees about the actions to be taken and everyone feels they are being understood in their needs. In order for this kind of communication to happen, these skills must be taught appropriately e continually, during the whole medical training. Up until now, there is no Brazilian instrument that fulfills this purpose. In Brazil, international guides are being used to achieve that. However, amongst the most internationally used guides and those most cited in specific literature, none is completely suitable to Brazil's reality. Objective: To compare four frequently used communications skills instruments, under the perspective of Brazilian Primary Care characteristics. Methodology: The guides were described, qualitatively analyzed and compared according to the following categories: guide structure, guide creation, important steps of the visit and focus on the doctor. Analysis: The instruments differ in easiness of use, focus in Primary Care teaching, discussion of important steps of the visit and focus on the doctor. They all discuss the construction of shared plans and none has a validated translation to Brazilian Portuguese. Conclusion: "A Consulta em 7 Passos" has the most suitable profile for Brazil's Primary Care, among the studied guides. However, the creation of a Brazilian guide is recommended to help structure, homogenize and facilitate the teaching of communication skills in Brazil's medical schools.

\section{INTRODUÇÃO}

A Atenção Primária à Saúde (APS) é a porta de entrada por onde as pessoas acessam um sistema de saúde estruturado em níveis de atenção. Ela se caracteriza também por um cuidado de saúde longitudinal, abrangente e coordenado com outros pontos do sistema, com atendimentos a quaisquer pessoas, independentemente de gênero, tipo de doença ou órgão acometido $^{1}$. Os profissionais da APS, especialmente médicos, têm diversos recursos para realizar o cuidado de saúde das pessoas, sendo o mais central a consulta médica.

Isto porque, além de ser a atividade com maior carga horária de trabalho desses profissionais ${ }^{2}$, é principalmente por meio da consulta que as pessoas atendidas nesse nível de atenção têm suas questões apreendidas por uma escuta qualificada. É o momento em que os profissionais, ao ouvirem as demandas das pessoas, têm a possibilidade de entender as necessidades delas. Dessa forma, podem estabelecer junto a elas planos de cuidado conjuntos que façam sentido tanto para o profissional quanto para a pessoa atendida, permitindo a resolução dessas questões. No espaço da consulta, um recurso importante é o uso de habilidades de comunicação. Em que pesem controvérsias sobre o uso do termo, optou-se por clas- sificar as habilidades de comunicação como uma tecnologia leve ${ }^{3}$, comumente utilizada na APS. Esta tecnologia tem a capacidade de aumentar a potência das consultas ${ }^{4}$ porque o uso de habilidades de comunicação está associado a melhor satisfação com a consulta, tanto para profissionais quanto para pessoas atendidas. Além disso, também aumenta a chance de descobrir os motivos que realmente trouxeram a pessoa à consulta $^{5}$ e facilita e melhora a relação entre profissional e pessoa atendida, aumentando a possibilidade de tornar este espaço de encontro mais resolutivo, já que está associado a aumento na adesão ao tratamento ${ }^{6}$ e melhora de sintomas . $^{7}$

Para que as consultas sejam mais potentes, é necessário que o uso das habilidades de comunicação seja feito de forma efetiva, que pode ser definida como a interação entre pessoas, profissionais de saúde e familiares de modo a realizar uma troca de informações em que todas as partes passem a ter o mesmo entendimento sobre a natureza do problema, acordem sobre as medidas a serem tomadas e se sintam acolhidas e entendidas em suas necessidades ${ }^{8-10}$.

Somente quando são ensinados de forma adequada é que estudantes e profissionais podem alcançar este tipo de comunicação. Esta adequação envolve também a oportunidade de 
refletir e discutir sobre suas ações entre si e com os facilitadores, de modo a permitir um desenvolvimento crítico dessas habilidades. Deve ocorrer, também, de forma longitudinal, permeando toda a formação médica, seja na graduação, residência médica ou na educação continuada dos profissionais. Além disso, deve se dar de forma estruturada, com o objetivo de manter alguma uniformidade no processo de ensino.

Diversos guias de comunicação foram criados, em serviços-escola, para padronizar e, assim, facilitar o ensino das habilidades de comunicação. A maioria deles foi criada por iniciativas de professores da área de comunicação ou humanidades médicas ou mesmo de departamentos de medicina de família e comunidades locais. A maior parte desses guias apresenta a mesma estrutura geral, já que busca ensinar e avaliar o uso de um núcleo comum de habilidades, passando por todas as etapas da consulta. No entanto, cada guia tem sua singularidade: seja em abordar habilidades em situações especiais $^{11}$, na forma de realizar as avaliações das habilidades ${ }^{12}$, nos requisitos para uso do guia ${ }^{13}$ ou mesmo na forma de dividir os tempos da consulta ${ }^{14}$. Essas diferenças têm origem também nas características dos serviços e cultura das localidades onde foram criados esses instrumentos. Isto acontece uma vez que foram pensados, inicialmente, para serem usados pelos estudantes e profissionais do local de origem.

No Brasil, o documento que normatiza as Diretrizes Curriculares do curso de graduação em Medicina de 2014 situa a habilidade em comunicação como uma das bases da formação do graduando ${ }^{15}$. Assim como no resto do mundo, o ensino dessas habilidades se dá de forma heterogênea. A grande maioria não tem disciplinas em seu currículo e utiliza o aprendizado prático por meio de exemplos (e da falta deles). Em algumas universidades, as habilidades de comunicação são desenvolvidas longitudinalmente durante o curso, sem, no entanto, ter um espaço específico ${ }^{16}$. Desta maneira, o uso de guias facilita e homogeneíza esse aprendizado.

Ao se pensar no ensino das habilidades de comunicação no cenário da APS brasileira, algumas considerações devem ser feitas na escolha do guia mais adequado. Os locais de origem dos guias mais comumente usados têm realidades muito diferentes da brasileira, o que impede a simples transposição dos guias. Outro ponto importante a ressaltar é que não existe um guia feito no Brasil que considere a prática da APS nacional. Por fim, as pequenas diferenças que esses instrumentos apresentam entre si é outro fator que dificulta a escolha certa de um guia mais adequado, já que não existe um instrumento considerado padrão.

Até o momento, são escassos os estudos que comparam os diferentes guias, permitindo encontrar um perfil de uso para cada situação específica. Este trabalho tem como objetivo estudar quatro principais guias e comparar suas características, levando em consideração o ensino de habilidades de comunicação na realidade da APS brasileira.

\section{MÉTODO}

Optou-se por um estudo teórico descritivo de quatro guias de habilidades de comunicação: “Guia para a Entrevista Médica de Calgary-Cambridge - Processo de Comunicação" (Calgary-Cambridge Guide to the Medical Interview - Communication Process) ${ }^{17}$, "A Consulta em 7 Passos"14, "MAAS-Global 2000"13 e "Estrutura SEGUE" (SEGUE Framework)12.

Os critérios de escolha dos guias foram: ampla utilização em processos de ensino e avaliação de habilidades de comunicação em todos os níveis da formação médica (graduação, residência e educação continuada) em diversos países, ampla citação em literatura internacional ${ }^{18}$, facilidade de uso entre profissionais e professores da área de comunicação em saúde e familiaridade do autor com os quatro guias.

Esses quatro guias foram submetidos a uma análise comparativa do tipo qualitativa. Em primeiro lugar, foram lidos exaustivamente e verificados quanto a aspectos descritivos relativos a origem, forma, conteúdo e estrutura dos instrumentos.

Dessa imersão no material empírico foram criadas as seguintes categorias analíticas: estrutura do guia, criação do guia, etapas importantes da consulta e foco no profissional.

\section{DESCRIÇÃO DOS GUIAS}

\section{Guias de Calgary-Cambridge}

Foram criados em 1996, como um projeto conjunto de Suzanne Kurtz, professora de comunicação nas faculdades de Medicina e Educação da Universidade de Calgary, Canadá, e Jonathan Silverman, médico de família, reitor clínico associado e diretor de estudos de comunicação na escola de Medicina da Universidade de Cambridge, Inglaterra. Tiveram suas primeiras versões lançadas em 1998, como livros.

Dividem-se em guia de Conteúdos de Comunicação, que discute uma aplicação da anamnese clínica tradicional, incluindo o ponto de vista da pessoa atendida, e guia de Habilidades Processuais de Comunicação, foco deste artigo, que discute a forma como ocorre esta comunicação, por meio de 71 habilidades principais.

Em 2003, como parte de uma evolução contínua, os guias foram melhorados com a publicação de novas versões, que se tornaram mais visuais e melhoraram a forma como as habilidades de comunicação são introduzidas e inseridas no currículo médico. 
O Guia de Habilidades Processuais de Comunicação de Calgary-Cambridge divide o espaço da consulta em cinco tarefas sequenciais e duas longitudinais, demonstradas na Figura 1.

Figura 1

Espaço da consulta segundo o Guia de Habilidades

Processuais de Comunicação de Calgary-Cambridge

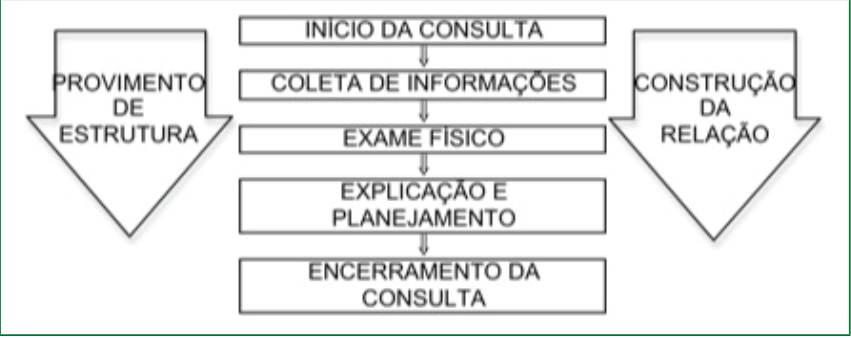

As etapas seguem a forma como estes passos de fato acontecem durante uma consulta. Exceto pelo exame físico, cada uma dessas tarefas tem uma série de habilidades de comunicação inclusas, que serão descritas sucintamente a seguir.

\section{Início da consulta}

Nesta tarefa, são enfocadas habilidades que concernem à preparação para a consulta, com o término de tarefas anteriores para focar na nova consulta e atenção para o conforto pessoal; o estabelecimento de uma relação inicial, levando em conta a forma como cumprimenta a pessoa, se apresenta e demonstra interesse no conforto dela; e a identificação dos motivos da consulta, com perguntas iniciais abertas, escuta ativa e negociação da agenda daquele encontro.

\section{Coleta de informações}

Nesta etapa, ocorre a exploração dos problemas do paciente, considerando a perspectiva tanto biomédica quanto a da pessoa atendida, utilizando-se de narrativas de sua parte, buscando suas ideias, preocupações, expectativas e efeitos em sua funcionalidade, uso de perguntas abertas e fechamento progressivo delas, respostas que facilitem que a pessoa continue seu relato, clarificação de frases que possam deixar dúvidas, resumos parciais do relato e uso de linguagem acessível e concisa, evitando jargões.

\section{Explicação e planejamento}

Este momento inicial da conclusão da consulta utiliza habilidades como o provimento de quantidades e tipos corretos de informação, separadas em "porções" pequenas; checa o entendimento da pessoa, questiona o conhecimento prévio de sua situação; usa técnicas para facilitar que a pessoa se lembre do que foi discutido, como repetições e resumos; usa métodos vi- suais; alcança um entendimento conjunto; relaciona a explicação ao contexto e forma de entendimento da pessoa acerca do problema; encoraja o saneamento de dúvidas; realiza um planejamento conjunto, envolvendo a pessoa, discutindo opções terapêuticas; negocia um plano e checa a aceitabilidade deste.

\section{Encerramento da consulta}

O encontro é finalizado por meio do acordo de planejamento futuro, como retornos, o que fazer caso algo não vá como esperado, além do uso de resumos gerais que implicam todo o encontro e da verificação se a pessoa está de acordo e confortável com o plano acordado.

\section{Construção da relação}

É uma das etapas longitudinais em que são importantes habilidades como o uso de linguagem não verbal adequada, observando seu uso por parte da pessoa atendida, uso de atitudes relacionais, como empatia, aceitação sem julgamento das percepções da pessoa, além do envolvimento da pessoa no processo de cuidado, como partilhar pensamentos, explicar a linha de raciocínio utilizada e as etapas do exame físico.

\section{Provimento de estrutura}

Durante toda a consulta, é importante estabelecer uma estrutura que evidencie a organização das etapas e do ritmo de consulta, utilizando-se resumos de cada questão discutida e sinalizações que demonstrem a progressão da consulta, além de manter uma sequência clara das etapas consecutivas.

\section{"A CONSULTA EM 7 PASSOS"}

O guia contido no livro de mesmo nome foi publicado em Lisboa, Portugal, em 2008 pelo médico de família Vitor Ramos.

Entende-se como um guia ou estrutura para balizar a realização de consultas por médicos de família. No entanto, se diz "uma proposta de trabalho", na qual o profissional pode colocar características e aprendizados pessoais em sua prática.

Foi criado inicialmente pela equipe do Centro de Saúde de Cascais, sendo que diversos médicos de família, internos e residentes de Medicina de Família e Comunidade, ligados à Escola Nacional de Saúde Pública da Universidade Nova de Lisboa, contribuiriam com suas visões.

Divide a consulta em três fases e em sete passos dentro dessas fases. A primeira fase, chamada fase inicial, corresponde à preparação e aos primeiros minutos da consulta. A segunda, a fase intermédia, abrange as etapas de exploração, avaliação e planejamento. A última, a fase final, se ocupa do encerramento e da reflexão final.

A seguir, cada um dos passos é descrito. 


\section{Preparação}

Momento primordial da consulta, quando o médico que vai iniciar o atendimento observa se três aspectos importantes estão adequados: o consultório, os dados anteriores da pessoa a ser atendida e a sua própria condição pessoal.

Avalia o registro anterior da pessoa, sua lista de problemas e últimos planos de cuidado, a fim de relembrar de quem se trata e observar se havia deixado alguma anotação ou plano para este novo encontro.

Além disso, checa condições do consultório, como limpeza, organização e presença de materiais adequados ao próximo atendimento.

Por fim, faz uma autoavaliação para saber o quão apto está para este novo encontro, como necessidades fisiológicas, estado emocional, problemas pessoais. Já que a interação é chave para o cuidado na atenção primária, as condições pessoais do médico devem, portanto, ser observadas.

\section{Os primeiros minutos}

No início da consulta, atenta-se para aspectos como a forma como o médico chama seu paciente, como o recebe em seu consultório, a forma como o cumprimenta.

Observam-se também facetas de comunicação, como expressões corporais, tom de voz e primeiras frases. Neste momento, o profissional deve fazer a escuta inicial da pessoa atendida, utilizando perguntas abertas para poder entender as demandas trazidas, eventuais demandas ocultas e pactuar quais questões serão abordadas neste encontro (delimitação de agenda da consulta) por meio de um resumo que clarifique esta primeira etapa da conversa.

\section{Exploração}

Realiza-se a anamnese, o exame objetivo e a contextualização dos problemas. Esta anamnese leva em conta o aspecto tanto biomédico quanto da experiência pessoal com a doença, que inclui sentimentos, expectativas, explicações, impactos na funcionalidade da pessoa.

Unida a um exame físico focado nos problemas e hipóteses consideradas, permite ao médico formar avaliações adequadas ao cuidado, que é o foco do Passo 4.

\section{Avaliação}

Na avaliação da situação de saúde, realiza-se um diagnóstico o mais preciso possível dos problemas apresentados, considerando aspectos de prognóstico e devolvendo à pessoa uma explicação adequada.

Nessa explicação, o médico deve levar em conta a linguagem utilizada, de forma que permita ao paciente entender que é uma explicação do problema que trouxe.
Para o prognóstico, cabe fazer um breve resumo com a pessoa de pontos negativos, positivos e fatores de proteção e de risco para cada um dos problemas, a fim de preparar para a realização dos planos, abordados no Passo 5.

\section{Plano}

Na realização dos planos de cuidado, é importante explicá-los à pessoa, justificando-os com base nas avaliações e prognósticos. Além disso, é primordial acordar com a pessoa quais deles são passíveis de ser realizados e aceitáveis, e quais precisam de adaptações.

Cabem também três aspectos importantes: o empoderamento das pessoas, por meio da promoção do autocuidado; a realização da proposta de seguimento, com planos de retorno e o que fazer caso haja algum problema; e, por fim, a proposição de medidas preventivas, sejam elas programáticas ou oportunísticas.

\section{Encerramento}

Verifica-se se ficou alguma dúvida, se os acordos foram entendidos e se a pessoa entendeu que seu problema foi encaminhado satisfatoriamente.

Por fim, é importante encerrar formalmente a consulta, indicando verbalmente este momento por meio de olhar ou cumprimento.

\section{Reflexão e notas finais}

Dedica-se ao momento imediatamente posterior ao término do encontro. Aqui cabe encerrar o registro adequado, seguindo o Registro Clínico Orientado a Problemas, pensar em pontos positivos e negativos da consulta e registrar notas prospectivas importantes para a continuidade do cuidado.

\section{"MAAS GLOBAL 2000"}

Esse instrumento, atualizado em 2000, foi criado por Jacques van Thiel e Paul Ram, médicos de família do Departamento de Medicina de Família da Universidade de Maastricht, Holanda, e Jan van Dalen, psicólogo do Laboratório de Habilidades da mesma universidade.

Usado no ensino de Medicina de Família da universidade desde 1992, seu nome deriva da sigla em holandês para Escore de Retirada de História e Aconselhamento de Maastricht, em tradução livre. Passou por diversas revisões até a versão de 2000.

Seu uso foi validado para ensino nos três níveis: graduação, residência médica e educação profissional continuada.

Divide a avaliação da consulta em 17 itens, subdivididos em três seções: habilidades de comunicação para cada fase se- 
parada; habilidades de comunicação gerais e aspectos médicos; e uma parte subsequente para outros feedbacks.

Cada um dos itens deve ser avaliado com pontuação de 0 (ausente) a 6 (excelente). Alguns itens podem ser avaliados como "n.a." quando não forem aplicáveis. As duas primeiras sessões têm subitens, que devem ser avaliados individualmente para compor a nota do item.

O manual deste guia inclui uma parte de definições de conceitos para clarificar sua avaliação, como resumos e habilidades de comunicação.

Ele define também condições da consulta para que seu uso resulte em avaliações ideais: a consulta deve ser relativamente completa e descomplicada, como quando o paciente tem somente uma queixa e a consulta passa por todas as fases. Em situações diferentes dessas, como várias queixas, mais de uma pessoa, consultas de retorno, deve-se definir previamente como será feita a avaliação.

A seguir, um resumo de cada seção:

\section{Habilidades de comunicação para cada fase separada}

Nesta seção, são avaliadas habilidades que definem cada passo da consulta. Essa avaliação prevê que as etapas estejam minimamente presentes, cabendo a avaliação da forma para a segunda seção e do conteúdo para a terceira.

Os itens de 1 a 7 são definidos por: introdução, consulta de seguimento, pedido de ajuda, exame físico, diagnóstico, manejo e avaliação da consulta.

São avaliadas nos subitens habilidades como: dar espaço para o paciente falar, questionar a razão da consulta, nomear queixas anteriores, questionar aderência a planos prévios, nomear demandas que trouxeram à consulta e por que veio neste momento, explicar o que vai ocorrer no exame físico, nomear achados e principais hipóteses, discutir alternativas terapêuticas e a factibilidade delas, questionar a opinião do paciente sobre a consulta e checar se a demanda foi atendida.

\section{Habilidades de comunicação gerais}

A seção seguinte avalia a forma como são aplicadas algumas habilidades de comunicação, que são entendidas como importantes e que podem ocorrer em diversas etapas da consulta.

Os itens de 8 a 13 são os seguintes: exploração, emoções, fornecimento de informações, resumos, estruturação e empatia.

São caracterizadas pela avaliação de: explorar a perspectiva do paciente sobre as informações dadas, responder a dicas não verbais, questionar sentimentos, refleti-los de forma adequada tanto em sua natureza quanto em intensidade, dar informações em pequenas porções e em linguagem acessível, realizar resumos com suas próprias palavras e checar enten- dimento, sequência lógica de passos da consulta, com balanceamento de tempo adequado entre os passos, expressão de empatia em respostas verbais e atitudes não verbais.

\section{Aspectos médicos}

A última seção avalia o conteúdo médico das habilidades de comunicação utilizadas. Deve ser avaliada tendo em mente protocolos estabelecidos para determinadas doenças e, quando não for este o caso, utilizando a melhor evidência existente.

Os itens de 14 a 17 são os de anamnese, exame físico, diagnóstico e manejo.

A ausência de subitens se deve à especificidade de cada consulta e ao fato de que alguns momentos da consulta não são firmemente ancorados em evidências.

\section{"ESTRUTURA SEGUE"}

Criado em 2001 por Gregory Makoul, foi a base para o ensino de habilidades de comunicação na Faculdade de Medicina da Universidade Northwestern, em Chicago, Estados Unidos.

Em 1992, pesquisas demonstraram que havia grande variação na qualidade e intensidade com que o ensino de habilidades de comunicação aparecia nas escolas norte-americanas. Partindo da necessidade de estabelecer um parâmetro para este ensino naquela universidade, foi iniciada a criação deste instrumento.

Em seu artigo referência, é demonstrada a experiência de criação, de uso para ensino das habilidades, bem como sua validação para avaliação e uso em pesquisas.

Divide a consulta em cinco partes, conforme o acrônimo SEGUE: arrumar o espaço (set the stage), extrair informação (elicit information), dar informação (give information), entender a perspectiva do paciente (understand the patient's perspective) e terminar o encontro (end the encounter), bem como uma última parte caso seja sugerido um novo plano ou modificado algum plano de tratamento ou prevenção. Esta sequência tem o objetivo de simular o fluxo habitual da consulta.

Cada um dos itens é nomeado em forma de tarefas, de forma a dar uma sensação de propósito ao estudante e permitindo que ele utilize diversas estratégias para completar a tarefa.

Entre as 25 tarefas das cinco divisões iniciais, 17 são de conteúdo de comunicação, sendo as outras oito processuais (marcadas com uma seta à). Para cada tarefa, há somente a possibilidade de marcar SIM ou NÃO. Algumas delas permitem a marcação como "n.a." (não se aplica), sendo somente possível nas de conteúdo.

Nas tarefas de conteúdo, deve ser marcado SIM quando o item aparece pelo menos uma vez na consulta, e NÃO se nunca aparecer. Nas processuais, inverte-se a lógica, pois se 
entende que estas atitudes devem acontecer o tempo todo da consulta para se considerar SIM.

Além da marcação dos itens, há um espaço para o preenchimento de feedbacks individuais, visando ao aprendizado do aluno, e não somente à soma de um escore.

A seguir, um pequeno resumo de cada uma das partes.

\section{Arrumar o espaço}

No início da consulta, são definidas tarefas como cumprimento adequado do paciente, definição de razão para o encontro, bem como a agenda para este, manutenção da privacidade e estabelecimento de uma relação.

\section{Extrair informação}

As tarefas processuais são: evitar perguntas fechadas, realizar escuta adequada e com tempo suficiente do paciente e checar o entendimento das informações colhidas. As de conteúdo englobam indagar questões físicas e psicossociais, entender aspectos subjetivos do adoecimento da pessoa e questionar histórico de saúde e hábitos de vida.

\section{Dar informação}

Avaliam-se tarefas de explicação de raciocínio diagnóstico e terapêutico, orientações sobre a situação da pessoa, bem como do funcionamento de seu corpo, encorajamento de perguntas e uso de linguagem adequada.

\section{Entender a perspectiva do paciente}

Esta parte inclui as tarefas de reconhecer os progressos e desafios da pessoa, tempo de espera, além da expressão de um tom respeitoso e empatia e preocupação com a pessoa.

\section{Terminar o encontro}

É importante revisar os próximos passos, além de checar se há algo mais a discutir.

\section{Caso haja algum plano terapêutico ou preventivo novo ou modificado}

Estas últimas sete tarefas objetivam avaliar a construção de planos de cuidado com a pessoa atendida, mediante a discussão de expectativas e metas dela em relação ao plano, envolvê-la nesta decisão, explicar benefícios, riscos e efeitos adversos do mesmo, instruí-la adequadamente sobre o plano, discutir possibilidade de seguir e a função da pessoa nele.

\section{ANÁLISE DESCRITIVA}

Na categoria Estrutura dos Guias, levamos em conta: a quantidade de itens avaliados (incluindo subitens), a maneira como as habilidades são apresentadas e como são avaliadas, se o guia avalia conteúdo e forma da comunicação juntos e se o guia requer alguma situação específica para utilização.

Quanto à quantidade de itens a serem avaliados (incluindo as subdivisões), todos os guias têm uma quantidade significativa de avaliações, com diferenças importantes entre eles, variando de 71 a 32 itens.

A forma de apresentação das habilidades é dicotomizada, seja por meio de tarefas ("Estrutura SEGUE" e "Calgary-Cambridge") ou de momentos ou passos ("MAAS Global 2000" e "A Consulta em 7 Passos"). Isto leva a avaliações também dicotomizadas, com os dois primeiros em avaliações de "sim" ou "não" para as tarefas, e os dois subsequentes por meio de escalas Likert ( 0 a 6, e 0 a 2, respectivamente).

Cabe salientar que ambas as escalas Likert têm número ímpar de itens, o que pode levar a um aumento do número de avaliações medianas (valores 1 e 3 , respectivamente), conforme Coelho ${ }^{19}$.

Sobre a discussão de forma e conteúdo de comunicação, inicialmente vale definir estes conceitos. Define-se conteúdo de comunicação como aquilo que as pessoas de fato dizem, as palavras que utilizam, a "substância" da informação que passam ou recebem (as perguntas que fazem, as demandas que abordam, os tratamentos que discutem). A forma de comunicação diz respeito a como o profissional interage com a pessoa atendida, de que maneira ele se relaciona com a informação que quer trocar, que habilidades (verbais ou não) ele usa ${ }^{11}$.

O "MAAS Global 2000" une conteúdo e forma no mesmo guia. $\mathrm{O}$ "Calgary-Cambridge" faz uma divisão entre habilidades de conteúdo e habilidades processuais (forma), dividindo-as em guias diferentes.

Sobre necessidades específicas para uso do guia, o "MAAS Global 2000" delimita critérios para uso, como a presença de somente uma queixa ou de uma consulta "completa e descomplicada".

Na categoria de Criação dos Guias, foram avaliados os profissionais que criaram os guias, se a APS é o objetivo de ensino, o idioma original do guia e a presença de validação ou tradução para o português brasileiro.

Quantos aos profissionais responsáveis pela criação deles, idealmente este grupo seria composto, mesmo que não exclusivamente, por médicos de família. Os guias de "Calgary-Cambridge", "A Consulta em 7 Passos" e o "MAAS Global 2000 " foram criados por equipes compostas, total ou parcialmente, por médicos de família.

O objetivo de ensino, também idealmente, seria a APS em qualquer nível de formação. Assim, os quatro guias foram criados para facilitar o ensino de habilidades de comunicação 
em diversos níveis. No entanto, somente "A Consulta em 7 Passos" e o "MAAS Global 2000" foram criados especificamente para o ensino dessas habilidades no contexto de APS.

Nenhum dos quatro guias tem uma versão validada para o português do Brasil. A “Estrutura SEGUE” tem uma versão traduzida para o português do Brasil por Mennin ${ }^{20}$,

e o "Calgary-Cambridge" por Miranda"21. "A Consulta em 7 Passos" é escrito em português de Portugal, o que facilita o entendimento, mas a diferença entre as duas línguas, apesar da mesma origem, pode levar a erros de interpretação.

$\mathrm{Na}$ avaliação de Etapas Importantes da Consulta, verificou-se a discussão de planos compartilhados, a presença de um olhar para a linguagem não verbal, a discussão de delimitação de agenda, a discussão de como proceder se algo sair diferente do esperado e a presença de foco para a estrutura da consulta.

A formulação de planos conjuntos, que aumenta a adesão e a efetividade destes ${ }^{11}$, também é comum na APS, e todos os guias citam esta etapa.

Quanto à linguagem não verbal, aspecto fundamental da comunicação em saúde ${ }^{23}$, o "MAAS Global 2000", " A Consulta em 7 Passos" e o "Calgary-Cambridge" dão atenção especificamente a esta forma. A "Estrutura SEGUE" a cita somente ao tratar de feedbacks não verbais às pessoas enquanto estão se expressando.

Na APS, nível de atenção que se caracteriza por consultas com motivos múltiplos e diversos, porém com o tempo como recurso e fator limitador, a delimitação da agenda desse encontro é fundamental para o bom andamento tanto da relação com a pessoa atendida quanto para o cuidado de saúde dela. Entende-se a delimitação de agenda da consulta como o acordo, preferencialmente verbal, entre profissional e a pessoa atendida, de quais demandas trazidas serão abordadas nesse encontro. Este acordo deve ser bilateral, pois ambos devem definir quais são as questões prioritárias e, destas, quais são viáveis para essa consulta ${ }^{22}$. Nestes termos, o "Calgary-Cambridge" e "A Consulta em 7 Passos" citam esta atitude em suas avaliações.

Outro recurso comum é estabelecer planos para quando algo que foi acordado não sair como planejado, como redes de proteção. Este recurso aparece no "Calgary-Cambridge" e "A Consulta em 7 Passos".

Dos quatro guias, somente o "Calgary-Cambridge" discute como tarefa específica o provimento de estrutura para a consulta. Embora todos caracterizem a consulta como uma sequência de passos, nenhum dos outros dá ênfase à estrutura como um passo ou tarefa a ser realizada.

Para a avaliação de Foco no Profissional, buscamos o fato de levar em conta aspectos pessoais do profissional e a dis- cussão sobre o registro do encontro. Definem-se aspectos pessoais, como nível de cansaço, estado emocional no momento da consulta, necessidades fisiológicas, bem como a influência da consulta anterior no profissional ${ }^{14}$, que aparecem nas avaliações de "A Consulta em 7 Passos" e "Calgary-Cambridge".

O registro clínico é uma ferramenta eficaz para alcançar um dos atributos principais da APS, a longitudinalidade ${ }^{1}$. Serve para a comunicação, tanto para outros profissionais que também cuidem daquela pessoa, quanto para si mesmo; serve como um registro ético e legal das discussões e condutas tomadas no encontro ${ }^{24}$, bem como para garantir a segurança da pessoa atendida, quanto aos seus cuidados de saúde. A discussão sobre este registro está presente em "A Consulta em 7 Passos".

O Quadro 1 apresenta um resumo das categorias avaliadas em cada guia.

\section{DISCUSSÃO}

Todos os guias utilizados neste estudo são bastante presentes na literatura sobre o tema ${ }^{18}$ e contribuíram significativamente para o ensino de habilidades de comunicação em seus locais de criação, bem como serviram de base para processos semelhantes em outros locais.

Este processo ainda é bastante incipiente na realidade de formação profissional brasileira, especialmente no ensino de APS, onde estas habilidades são de especial importância.

Devido à ausência de um guia nacional, entendeu-se a necessidade de avaliar alguns dos mais utilizados internacionalmente entre os existentes.

Em nossa análise para avaliar os instrumentos, observou-se que diferem em tamanho, abordagem de alguns pontos, origem, forma e condições de utilização. Algumas destas diferenças são mais importantes quando se tem em mente a escolha de um instrumento específico para o ensino na APS brasileira.

Inicialmente, a quantidade de itens a ser avaliada é o primeiro ponto sensível. $\mathrm{O}$ "Calgary-Cambridge" tem uma quantidade muito grande de itens (71), mais que o dobro da "Estrutura SEGUE" (32). Entende-se que a tarefa de avaliar/ ensinar comunicação numa consulta é altamente complexa. No entanto, o instrumento deve ser enxuto o suficiente para que se torne prático para avaliar consultas que, neste contexto, duram aproximadamente 15 minutos $^{25}$.

Sobre as diferentes abordagens dos guias, ressaltam-se alguns pontos de importância na APS brasileira. Criar planos compartilhados é uma atitude importante quando consideramos o ensino na APS, embora isto deva ser abordado em qualquer realidade de saúde, independentemente do nível de 


\begin{tabular}{|c|c|c|c|c|}
\hline \multicolumn{5}{|c|}{$\begin{array}{c}\text { QUADRO } 1 \\
\text { Resumo das categorias avaliada }\end{array}$} \\
\hline & $\begin{array}{l}\text { Calgary- } \\
\text { Cambridge }\end{array}$ & $\begin{array}{l}\text { A Consulta } \\
\text { em } 7 \text { Passos }\end{array}$ & MAAS Global 2000 & Estrutura SEGUE \\
\hline \multicolumn{5}{|l|}{ Estrutura do guia } \\
\hline Une conteúdo e forma & $\begin{array}{l}\text { Não (guias } \\
\text { separados) }\end{array}$ & Não & Sim & Não \\
\hline Apresentação das habilidades de comunicação & Tarefas & Passos & Passos & Tarefas \\
\hline Forma de avaliação das habilidades & Sim ou Não & Likert (0 a 2) & Likert (0 a 6) & Sim ou Não \\
\hline Quantidade de itens avaliados (incluindo subitens) & 71 & 52 & 47 & 32 \\
\hline Requer situação específica para uso & Não & Não & Sim & Não \\
\hline \multicolumn{5}{|l|}{ Criação do guia } \\
\hline Idioma original & Inglês & $\begin{array}{l}\text { Português } \\
\text { (Portugal) }\end{array}$ & Holandês & Inglês \\
\hline Tradução para o português (Brasil) & $\operatorname{Sim}$ & Não & Não & $\operatorname{Sim}$ \\
\hline Validado em português (Brasil) & Não & Não & Não & Não \\
\hline Equipe de criação & Contém MFCs & Contém MFCs & Contém MFCs & Não contém MFCs \\
\hline APS como objetivo de ensino & Não & Sim & Sim & Não \\
\hline \multicolumn{5}{|l|}{ Etapas importantes da consulta } \\
\hline Importância para a estrutura da consulta & Sim & Não & Não & Não \\
\hline Discute delimitação de agenda & Sim & Sim & Não & Não \\
\hline Discute planos compartilhados & $\operatorname{Sim}$ & $\operatorname{Sim}$ & Sim & $\operatorname{Sim}$ \\
\hline Discute conduta se algo sair errado & Sim & Sim & Não & Não \\
\hline Importância para linguagem não verbal & $\operatorname{Sim}$ & Sim & $\operatorname{Sim}$ & Indiretamente \\
\hline \multicolumn{5}{|l|}{ Foco no profissional } \\
\hline Leva em conta aspectos pessoais do profissional & $\operatorname{Sim}$ & Sim & Não & Não \\
\hline Discussão sobre registro da consulta & Não & Sim & Não & Não \\
\hline
\end{tabular}

atenção. Entendendo-se que a participação da pessoa atendida é primordial na delimitação dos planos de cuidado, os quatro guias abordam esta tarefa.

Neste sentido, a delimitação de agenda permite que ambas as partes definam quais demandas podem ser e serão abordadas naquele encontro. Neste nível de atenção, a quantidade de queixas entre as consultas varia muito: há encontros com somente uma demanda, outros com inúmeras, e alguns com somente uma demanda inicial, que se coloca à frente de diversas outras que a pessoa entende ser melhor não dizer no primeiro momento, mas que a incomodam de forma importante. A este último caso chamamos demanda oculta ${ }^{22}$. "A Consulta em 7 Passos" e o "Calgary-Cambridge" pontuam esta atitude na consulta.

Além disso, a discussão da importância de se prover estrutura na consulta tem grande peso em um nível de atenção em que as consultas têm demandas e propósitos muito diferentes entre si. $\mathrm{O}$ fato de se estabelecer uma estrutura que não seja excessivamente rígida e que dê apoio e segurança tanto ao profissional quanto à pessoa atendida facilita muito o aprendizado de como fazer uma consulta e como usar as habilidades de comunicação nela. $\mathrm{O}$ "Calgary-Cambridge" discorre sobre isto como uma tarefa especificamente.

Por fim, o registro clínico é uma ferramenta fundamental na APS. O foco neste registro, como um dos passos dos guias, permite à pessoa em formação a melhor compreensão desta importância e a habitua a realizá-lo de forma adequada, foco que aparece somente em "A Consulta em 7 Passos".

Quanto à origem dos guias, alguns aspectos são pontuados. Eles foram pensados para facilitar o ensino das habilidades de comunicação nos diversos momentos do ensino médico. Seja como aluno de graduação ou profissionais ex- 
perientes, atentar para as necessidades físicas ou emocionais do profissional permite um aprendizado mais tranquilo, humanizado e, portanto, mais efetivo. Este olhar aparece em "A Consulta em 7 Passos" e "Calgary-Cambridge".

Ainda, um guia que servisse idealmente para o ensino de APS no Brasil deveria ter sido criado por médicos de família, ao menos em parte, e ser voltado ao ensino de APS. O "MAAS Global 2000" e "A Consulta em 7 Passos" têm essas duas características a seu favor.

Ainda levando em conta a APS brasileira, é preciso considerar a questão da língua para o uso corrente do guia. $\mathrm{Ne}-$ nhum deles tem uma versão validada para o português do Brasil. O "Calgary-Cambridge" e a "Estrutura SEGUE" têm versões traduzidas. Dessa forma, qualquer um dos guias que fosse escolhido em nosso país teria que ser usado em seu idioma original, em língua inglesa (o "MAAS Global 2000" tem uma versão validada para o inglês) ou numa versão traduzida, mas não validada. Em qualquer uma das situações, haveria perdas na interpretação.

Por fim, considerando as condições de uso, o "MAAS Global 2000" especifica critérios, o que prejudica de forma importante seu uso na APS brasileira (e talvez em qualquer realidade), já que este nível de atenção é caracterizado por diversidade de demandas em quantidades variáveis e consultas bastante diferentes entre si. Além disso, mesmo que fossem especificados critérios para adaptar seu uso a cada consulta diferente, isto implicaria um tipo de avaliação diferente para cada momento, o que comprometeria a validade interna da avaliação e a reprodutibilidade do instrumento.

O Quadro 2 apresenta um resumo da avaliação das categorias de análise dos quatro guias.

\begin{tabular}{|c|c|c|c|c|}
\hline & Resumo qualitati & $\begin{array}{c}\text { QUADRo } 2 \\
\text { vo da avaliação das categ }\end{array}$ & yorias de análise & \\
\hline Categoria & Calgary-Cambridge & A Consulta em 7 Passos & MAAS Global 2000 & Estrutura SEGUE \\
\hline Estrutura do guia & $\begin{array}{c}++ \\
\text { Grande quantidade de } \\
\text { itens a avaliar }\end{array}$ & $\begin{array}{c}++ \\
\text { Grande quantidade de } \\
\text { itens a avaliar }\end{array}$ & $\begin{array}{c}+ \\
\text { Requer situações } \\
\text { específicas para uso }\end{array}$ & $\begin{array}{l}+++ \\
\text { Poucos itens avaliados } \\
\text { facilitam o uso }\end{array}$ \\
\hline Criação do guia & $\begin{array}{c}+ \\
\text { Tradução não validada, não } \\
\text { tem como alvo APS }\end{array}$ & $\begin{array}{l}+++ \\
\text { Sem tradução, mas em } \\
\text { idioma próximo, alvo de } \\
\text { ensino na APS }\end{array}$ & $\begin{array}{l}+ \\
\text { Sem tradução, alvo de } \\
\text { ensino na APS }\end{array}$ & $\begin{array}{c}+ \\
\text { Tradução não validada, não } \\
\text { tem como alvo APS }\end{array}$ \\
\hline $\begin{array}{l}\text { Etapas importantes da } \\
\text { consulta }\end{array}$ & $\begin{array}{c}+++ \\
\text { Discute passos } \\
\text { significativos da consulta }\end{array}$ & $\begin{array}{c}++ \\
\text { Não discute provimento de } \\
\text { estrutura }\end{array}$ & $\begin{array}{l}+ \\
\text { Não discute provimento de } \\
\text { estrutura, nem delimitação } \\
\text { de agenda }\end{array}$ & $\begin{array}{l}+ \\
\text { Não discute provimento de } \\
\text { estrutura, nem delimitação } \\
\text { de agenda }\end{array}$ \\
\hline Foco no profissional & $\stackrel{++}{++}$ Não discute registro clínico & $\begin{array}{l}+++ \\
\text { Foco no profissional }\end{array}$ & $\stackrel{+}{\text { Sem foco no profissional }}$ & $\stackrel{+}{\text { Sem foco no profissional }}$ \\
\hline
\end{tabular}

\section{CONCLUSÃO}

Todos os guias apresentados neste estudo têm potencialidades e pontos frágeis quando se pensa em qual guia escolher para o ensino na APS brasileira. Nenhum deles tem todas as características adequadas para este uso.

Considerados os principais pontos importantes nos guias, temos como opções talvez mais adequadas à realidade brasileira na APS os guias de "Calgary-Cambridge" e "A Consulta em 7 Passos". Ambos têm como ponto de fragilidade a questão do idioma. A questão do tamanho também é significativa em ambos.

Ao fim desta análise, considerados os pontos negativos, a melhor opção, do ponto de vista dos autores do presente artigo, é "A Consulta em 7 Passos".

No entanto, a opção ideal para potencializar o ensino de habilidades de comunicação na APS brasileira seria a criação de um guia nacional, que levasse em conta as características mais centrais deste nível de atenção e da realidade local.

\section{REFERÊNCIAS}

1. Starfield B. Is primary care essential? Lancet. 1994;344(8930):1129-33.

2. Kurtz S, Silverman J, Draper J. Teaching and Learning Communication Skills in Medicine. 2nd ed. Oxford, England: Radcliffe Publishing; 2005.

3. Merhy EE. Saúde: a cartografia do trabalho vivo. $4^{a}$ ed. São Paulo: Hucitec; 2005.

4. Rider EA, Keefer CH. Communication skills competencies: definitions and a teaching toolbox. Med Educ. 2006;40(7):624-9.

5. Langewitz W, Denz M, Keller A, Kiss A, Ruttimann S, Wossmer B. Spontaneous talking time at start of 
consultation in outpatient clinic: cohort study. BMJ. 2002;325(7366):682-3.

6. Jansen KL, Rosenbaum ME. The State of Communication Education in Family Medicine Residencies. Fam Med. 2016;48(6):445-51.

7. Stewart M, Brown JB, Donner A, McWhinney IR, Oates J, Weston WW, et al. The impact of patient-centered care on outcomes. J Fam Pract. 2000;49(9):796-804.

8. Rios IC. Comunicação em Medicina. Revista de Medicina. 2012;91(3):4.

9. Maguire P, Pitceathly C. Key communication skills and how to acquire them. BMJ. 2002;325(7366):697-700.

10. Van Dalen J, Bartholomeus P, Kerkhofs E, Lulofs R, Van Thiel J, Rethans JJ, et al. Teaching and assessing communication skills in Maastricht: the first twenty years. Med Teach. 2001;23(3):245-51.

11. Silverman J, Kurtz S, Draper J. Skills for Communicating with Patients. 3rd ed. Oxford, England: Radcliffe Pub; 2016.

12. Makoul G. The SEGUE Framework for teaching and assessing communication skills. Patient Educ Couns. 2001;45(1):23-34.

13. Van Thiel J, Ram P, Van Dalen J. MAAS-Global Manual. Maastricht, Netherlands: Maastricht University; 2000.

14. Ramos V. A consulta em 7 passos: Execução e análise crítica de consultas em medicina geral e familiar. Lisboa: VFBM Comunicação; 2008.

15. Brasil. Ministério da Educação. Resolução no 3, de 20 de junho de 2014. Institui Diretrizes Curriculares Nacionais do Curso de Graduação em Medicina e dá outras providências. Brasília: Diário Oficial da União; 2014.

16. Varga CRR, Almeida VC, Germano CMR, Melo DG, Chachá SGF, Souto BGA, et al. Relato de experiência: o uso de simulações no processo de ensino-aprendizagem em medicina. Revista Brasileira de Educação Médica. 2009;33:291-7.

17. Kurtz S, Silverman J, Benson J, Draper J. Marrying content and process in clinical method teaching: enhancing the Calgary-Cambridge guides. Acad Med. 2003;78(8):802-9.

18. Schirmer JM, Mauksch L, Lang F, Marvel MK, Zoppi K, Epstein RM, et al. Assessing communication competence: a review of current tools. Fam Med. 2005;37(3):184-92.

19. Coelho PS, Esteves SP. The choice between a 5-point and a 10-point scale in the framework of customer satisfaction measurement. [dissertação]. Lisboa: Instituto Superior de Estatística e Gestao de Informaçao - Universidade Nova de Lisboa; 2007.

20. Makoul G. A guide for instructor and learners for teaching and assessing communication skills. The SEGUE framework. Fortaleza: UFCE; 2005.

21. Miranda AAA. Habilidades de comunicação profissional/ paciente no processo de seleção e adaptação de aparelhos de amplificação sonora individuais: avaliação de uma estratégia de ensino [tese]. Bauru (SP): Universidade de São Paulo; 2015.

22. Carrió FB. Entrevista Clínica: Habilidades de Comunicação para Profissionais de Saúde. Porto Alegre: Artmed; 2009.

23. Silva LMG, Brasil VV, Guimarães HCQCP, Savonitti BHRdA, Silva MJP. Comunicação não-verbal: reflexões acerca da linguagem corporal. Revista Latino-Americana de Enfermagem. 2000;8:52-8.

24. Conselho Federal de Medicina. Resolução CFM $n^{\circ}$ 1.931/2009. Aprova o Código de Ética Médica. Brasília: CFM; 2010.

25. Vidal TB. O acesso avançado e sua relação com o número de atendimentos médicos em atenção primária à saúde [tese]. Porto Alegre: Universidade Federal do Rio Grande do Sul; 2013.

\section{CONTRIBUIÇÃO DOS AUTORES}

Carlos Frederico Confort Campos: análise e discussão dos dados, escrita e publicação do artigo. Izabel Cristina Rios: supervisão na análise e discussão dos dados, escrita e publicação do artigo.

\section{CONFLITO DE INTERESSES}

Os autores declaram não haver qualquer conflito de interesse.

\section{ENDEREÇO PARA CORRESPONDÊNCIA}

Carlos Frederico Confort Campos

Laboratório de Humanização

Rua Teodoro Sampaio, 115 - 1ํAㄱ An

Cerqueira César, São Paulo - SP

CEP: 05405-000

Email: cafred@gmail.com 